\title{
Efectos clínicos del pedaleo pasivo en personas con lesión medular torácica crónica
}

\author{
Clinical effects of passive leg cycling after \\ chronic thoracic spinal cord injury
}

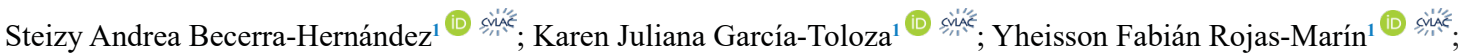

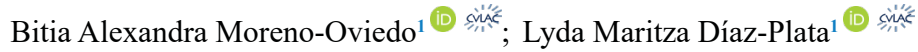 \\ *1mdiazpl@uis.edu.co
}

Forma de citar: Becerra Hernández SA, García Toloza KJ, Rojas Marín YF, Moreno Oviedo BA, Díaz Plata LM. Salud UIS. 2022; e22011. doi: https://doi.org/10.18273/saluduis.54.e:22011 @c) (i)

\begin{abstract}
Resumen
Introducción: el pedaleo pasivo (PP) es considerado una herramienta en la rehabilitación de personas con lesión medular (LM). Objetivo: revisar la evidencia científica sobre los efectos clínicos producidos por intervenciones que emplean PP en personas con lesión medular torácica crónica. Metodología: revisión narrativa. Se realizó una búsqueda en las bases de información científica MEDLINE, CINAHL, LILACS, PEDro y Clinicalkey de artículos en inglés y español, con términos como "spinal cord injury", "thoracic injuries", "passive pedaling", "cycling passive" y "protocol", entre otros. Resultados: se obtuvieron 14 artículos que describen efectos limitados a nivel de los sistemas cardiovascular, neuromuscular y musculoesquelético; se encontró heterogeneidad clínica en los sujetos del estudio, así como diferencias en los parámetros de realización del pedaleo. Conclusiones: los estudios del PP en personas con LM torácica crónica evalúan en su mayoría el efecto de una única sesión. Los cambios cardiovasculares son estudiados con mayor frecuencia, aunque se requieren más investigaciones.
\end{abstract}

Palabras clave: Lesiones de la médula espinal; Pedaleo pasivo; Lesiones torácicas.

\begin{abstract}
Introduction: Passive leg cycling is considered as a tool in rehabilitation of people with spinal cord injury (SCI). Objective: To review the scientific evidence on the clinical effects produced by interventions that use passive leg cycling in people with chronic thoracic spinal cord injury. Methodology: Narrative review. A search was performed by using the MEDLINE, CINAHL, LILACS, PEDro and Clinicalkey databases, for articles in English and Spanish,
\end{abstract}

\footnotetext{
${ }^{1}$ Universidad Industrial de Santander. Bucaramanga, Colombia
} 
with terms such as "spinal cord injury", "thoracic injuries", "passive pedaling", "passive cycling" and "protocol”, among others. Results: 14 papers were included in our research which described limited effects at the level of the cardiovascular, neuromuscular and musculoskeletal systems. We found clinical heterogeneity in the subjects, as well as differences in the cycling parameters used by authors. Conclusions: Studies of PP in people with chronic thoracic SCI mostly evaluated the effect of a single session. Cardiovascular changes were mostly studied; however, more evidence is needed.

Keywords: Spinal cord injuries; Passive cycling; Thoracic injuries.

\section{Introducción}

El concepto de lesión medular (LM) incluye cualquier daño que produce un cambio temporal o permanente en la función de la médula espinal, sea motora, sensorial o autonómica, y, por tanto, puede resultar en disfunción de múltiples órganos ${ }^{1}$. La LM interrumpe, de manera completa o incompleta, las vías ascendentes y descendentes que transportan información entre el cerebro y la médula espinal, y provoca un compromiso clínico y funcional variable que depende del nivel y la extensión de la lesión ${ }^{2,3}$. Cuanto más alta sea la lesión, mayor será la afectación de la persona ${ }^{1}$. La Asociación Americana de Lesiones Medulares (American Spinal Injury Association - ASIA, por sus siglas en inglés) establece cinco grados de lesión (A hasta E) determinados por la ausencia o preservación de la función motora y sensitiva; la A es la de mayor compromiso clínico (LM completa) y la E, la de menor afectación (con función sensorial y motora normal) ${ }^{4}$.

La incidencia mundial de la LM varía entre 13 y 163 casos nuevos, y su prevalencia, entre 367 y 526 casos por cada 1000000 de personas por año. Esta lesión es más frecuente en hombres que en mujeres $(1,1$ a 7,6 veces) y su rango medio de edad puede variar: es más estrecho y a edades adultas más tempranas (3046 años) en los países menos desarrollados. Para el caso de Colombia, las causas de LM más comunes son las heridas por armas de fuego y cortopunzante y los accidentes de tránsito ${ }^{6}$.

Sumada a las deficiencias primarias generadas por una LM, la presencia de complicaciones médicas es común y tiende a aumentar con la edad y a estar asociada con un aumento del riesgo para enfermedad cardíaca $(\mathrm{OR}=2,72$; intervalo de confianza al $95 \%$ (IC95\%): $1,94-3,82)$ y accidente cerebrovascular $(\mathrm{OR}=3,72$; IC95 \%: 2,22-6,23) en comparación con personas sanas 7. Por otro lado, estas personas también pueden manifestar dolor nociceptivo o neuropático, úlceras por presión, complicaciones urinarias, trombosis venosa profunda, embolia pulmonar y alteraciones osteoarticulares, neuromusculares, gastrointestinales y psiquiátricas $^{8}$.
Las lesiones en niveles de T1 a T12 pueden asociarse con complicaciones respiratorias como atelectasia $(36,4 \%)$, neumonía $(31,4 \%)$ e insuficiencia respiratoria $(22,6 \%)^{9}$. Es importante considerar que algunas de las complicaciones están relacionadas directamente con la inactividad muscular derivada de la parálisis de los miembros inferiores.

Debido a esto, con el fin de aumentar la movilidad de las extremidades inferiores y disminuir o retrasar la aparición de algunas de estas complicaciones, se han desarrollado investigaciones centradas en diversas intervenciones terapéuticas ${ }^{10}$.Una de estas es el pedaleo pasivo (PP), técnica que consiste en un movimiento sincrónico de las extremidades realizado por un motor; es usada principalmente en personas con limitaciones en la movilidad, ya que este tipo de pedaleo no requiere control volitivo ${ }^{11}$. Estos movimientos pasivos podrían contribuir a conservar la movilidad articular, mejorar la mecánica circulatoria y conservar las propiedades físicas de cartílagos, músculos y ligamentos ${ }^{12,13}$. Sin embargo, la evidencia disponible para apoyar el uso de PP como tratamiento estándar en personas con LM es aún limitada y contradictoria ${ }^{14}$.

Este estudio tiene como objetivo revisar la evidencia científica sobre los efectos clínicos producidos por intervenciones que emplean PP en personas con LM crónica de nivel torácico en los sistemas cardiovascular, neuromuscular y musculoesquelético; asimismo, se busca revisar los parámetros que se establecen en los protocolos que guían su aplicación a fin de que puedan servir como referente para el planteamiento de futuros estudios experimentales que determinen su efecto en la salud de estas personas, minimicen los costos derivados de la atención médica de las complicaciones y mejoren su calidad de vida.

\section{Metodología}

Esta es una revisión narrativa de literatura científica con una aproximación a la metodología sistemática en términos de selección de las fuentes de información. En el período comprendido entre febrero y julio de 2020, se 
realizó una búsqueda bibliográfica en las bases de datos MEDLINE, CINAHL, LILACS, PEDro y Clinicalkey.

Los términos utilizados en este trabajo para labúsqueda de referencias bibliográficas fueron spinal cord injury, thoracic injuries, passive pedaling, cycling passive, protocol, lesión medular, lesión torácica, pedaleo pasivo, ciclismo pasivo y protocolo. Se incluyeron artículos originales en inglés o español, publicados entre 2000 y 2020, que usaban el PP de extremidades inferiores en personas con LM torácica mayor a 6 meses de evolución y clasificación ASIA A, B o C.

Para esta revisión se excluyeron aquellas investigaciones que empleaban el pedaleo con activación muscular voluntaria o eléctrica, por ejemplo, las que evaluaban las ventajas de la estimulación eléctrica funcional (FES cycling) o la estimulación eléctrica neuromuscular. No hubo criterios de exclusión relacionados con grupo de edad o sexo.

\section{Resultados}

De un total de 127 artículos encontrados durante la búsqueda usando las palabras clave, 11 fueron considerados elegibles. Adicionalmente, otras 3 publicaciones fueron identificadas a partir de una estrategia de revisión de sus referencias bibliográficas; finalmente, se obtuvieron 14 trabajos para el análisis: 12 experimentales y 2 revisiones. La Figura 1 muestra un resumen del proceso de selección.

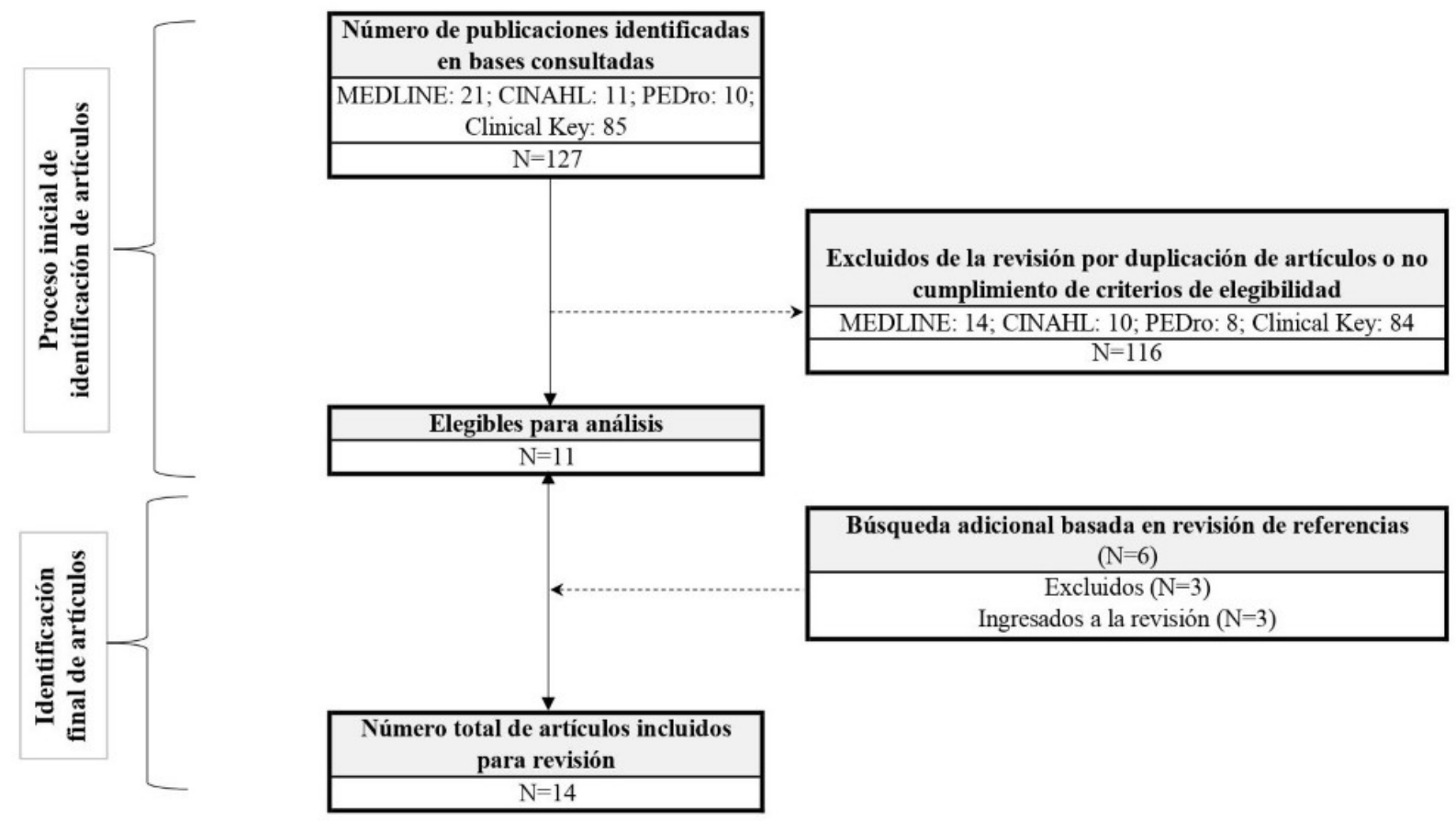

Figura 1. Diagrama de selección de artículos de la revisión narrativa

Aspectos generales de los protocolos de PP en los estudios revisados: para la aplicación del PP en personas con LM torácica, los autores de las investigaciones establecieron, en términos generales, que los sujetos de los estudios tuviesen la columna vertebral estable y contaran con rangos de movilidad articular en cadera y rodilla que permitieran la realización del patrón de pedaleo.

Adicionalmente, los participantes no debían presentar antecedentes de tabaquismo, úlceras por presión, trombosis venosa profunda e historia de enfermedad cardíaca, metabólica o pulmonar al momento de la realización del estudio. Previo a la intervención, en algunos estudios, los autores solicitaban a los participantes abstenerse del consumo de cafeína, alcohol o nicotina en las 12 horas anteriores.

En general, no se encontró una descripción detallada de las características biomecánicas que influyen en el pedaleo, como altura del asiento, longitud del brazo de la biela, posición del pie, distancia entre el pedaleador 
y la silla ${ }^{15}$. La cadencia (número de pedaleos por minuto) y el tiempo de duración de la sesión fueron los únicos parámetros mencionados consistentemente en los estudios. La cadencia reportada se estableció entre 15 y 70 revoluciones por minuto (rpm); 40 fue la más empleada. El rango del número de sesiones estuvo entre 1 y 36, mientras que el de la duración de la sesión estuvo entre 7 y 90 minutos.

\section{Efectos del pedaleo pasivo}

Las investigaciones que evalúan las consecuencias fisiológicas del PP en personas con LM torácica crónica se han centrado en los efectos a nivel de los sistemas cardiovascular, neuromuscular y musculoesquelético.

Efectos sobre el sistema cardiovascular central: en un estudio experimental ${ }^{16}$ en 11 participantes ( 5 personas sanas control y 6 con LM con nivel T8 a L1) se encontró que, posterior a una sesión de 6 minutos de PP y a una cadencia de $40 \mathrm{rpm}$, el gasto cardiaco y el volumen sistólico incrementaron en ambos grupos. En el grupo control, la frecuencia cardíaca (FC) se elevó al inicio del PP y después volvió lentamente al nivel de reposo hasta el final de este. En contraste, el grupo LM mostró cambios más pequeños en FC durante el PP, en comparación con el sano.

Estos hallazgos concuerdan con los resultados en los efectos obtenidos también por Muraki et al. ${ }^{17}$ en los volúmenes de eyección y FC, en una sesión de PP de 15 minutos de duración con incremento gradual de las rpm. En los primeros 5 minutos, la cadencia aumentó hasta alcanzar $20 \mathrm{rpm}$; los volúmenes de eyección y FC se elevaron y se mantuvieron durante esta etapa. Estos valores aumentaron gradualmente de manera proporcional a la cadencia de pedaleo que avanzó de forma progresiva hasta los últimos 10 minutos y terminó en una frecuencia de $70 \mathrm{rpm}$.

Efectos sobre el sistema cardiovascular periférico: en términos generales, se ha descrito que el movimiento de pedaleo es el medio más eficiente para mejorar el retorno venoso. Durante el PP de las extremidades inferiores, el lecho vascular plantar se comprime en cada revolución del pedal. La presión ejercida por el peso de la pierna en el pedal aumenta durante el movimiento ascendente del mismo. Este movimiento rítmico provoca una actividad similar a la de la bomba muscular durante la contracción ${ }^{18}$.

En un estudio en el cual participaron 15 sujetos con $\mathrm{LM}^{19}$ se encontró que posterior a una sesión de 10 minutos de PP a $40 \mathrm{rpm}$ en posición sedente se presentaba un aumento de la velocidad máxima y media del flujo sanguíneo en los músculos de las extremidades inferiores en $20 \%$ y $31 \%$, respectivamente ( $<0,01)$, y una disminución de la resistencia vascular periférica en un $11 \%(\mathrm{p}<0,05)$.

Sin embargo, Groothuis et al. ${ }^{20}$ sugirieron que los resultados del estudio anterior pudieron relacionarse con el momento de medición de la velocidad de los glóbulos rojos y la postura en que era ubicado el participante durante esta. La medición se realizaba a los 7 segundos de finalizada la intervención, y, para ello, el espaldar del asiento en el que se encontraba el participante con el tronco erguido se inclinaba $150^{\circ}$, lo que provocaba un cambio en la distribución de la sangre, que conducía a un aumento instantáneo (en 10 segundos) del retorno venoso, el volumen sistólico, el gasto cardíaco y la presión arterial. El aumento, por lo tanto, observado en la velocidad de los glóbulos rojos, encontrado en el estudio de Ballaz, se podría explicar por el cambio en la posición del cuerpo más que por el ciclismo pasivo de las piernas.

Ese análisis, se encuentra en línea con los hallazgos de Ter Woerds et $a l .^{21}$, quienes encontraron que el flujo sanguíneo en la arteria femoral común no cambió durante o después de 2 intervenciones (movimientos pasivos y PP). Los grupos experimental (8 personas con LM) y control ( 8 personas sin lesión) recibieron la misma intervención, que incluía, una sesión de movimientos pasivos en miembros inferiores realizados en supino y, posterior 20 minutos de descanso, PP a 35 rpm en posición sedente durante 20 minutos, seguido de un período de recuperación de 10 minutos; se concluyó que el PP no afectaba a la circulación periférica y, por lo tanto, no mejoraba la perfusión tisular.

Sin embargo, en un ensayo clínico controlado realizado un año después, también por Ballaz et al. ${ }^{18}$, en 17 sujetos con paraplejia, de los cuales 13 presentaban LM (8 sujetos asignados al grupo control y 9 al experimental), se encontraron resultados similares a su estudio de 2007. El grupo experimental que participó de un programa de PP en casa, a una frecuencia de $40 \mathrm{rpm}, 30$ minutos al día, 6 veces a la semana durante 6 semanas, presentó un aumento significativo de los valores postintervención en la velocidad media del flujo sanguíneo $(6,7 \pm 3$ vs $9,7 \pm 3,2$ $\mathrm{cm} / \mathrm{s} ; \mathrm{p}<0,01)$; no se observaron cambios en el grupo de control $(\mathrm{p}=0,68)$, quienes no recibieron intervención y permanecieron con sus rutinas habituales en casa. De la misma manera, la rigidez arterial postejercicio fue significativamente menor después del entrenamiento en el grupo experimental $(1,20 \pm 0,20$ vs $1,03 \pm 0,23$; 
$\mathrm{p}=0,04)$, mientras que no se observaron cambios en el grupo de control $(\mathrm{p}>0,05)$.

Efectos sobre el sistema neuromuscular: el efecto del PP sobre la espasticidad ha sido la principal variable analizada a nivel neuromuscular, se hallaron resultados contradictorios y es así como Kakebeeke et al. ${ }^{22}$, en un estudio experimental con 10 participantes con lesión a nivel de C6-T12, no encontraron diferencias significativas en la espasticidad medida a través de un dinamómetro isocinético posterior a una intervención que consistió en una sesión de PP de 30 minutos a una frecuencia de $40 \mathrm{rpm}$. Sin embargo, 6 de los 10 participantes, al preguntarles: ¿Usted piensa que está más, menos o igual de espástico?, informaron una reducción subjetiva de la espasticidad después de finalizada la sesión. Este efecto positivo según los autores podría atribuirse a una reducción de la espasticidad enel tronco.

Por otro lado, Krause et al..$^{23}$ publicaron un ensayo clínico cruzado en el que 5 participantes con LM a nivel de T3-T7 recibieron una sesión de FES entre 60 y 100 minutos (frecuencia de pedaleo no especificada) y una sesión de 30 minutos de PP, e informaron una disminución de la espasticidad inmediatamente después de la intervención con PP medida con el índice de relajación de la prueba del péndulo de Wartenberg, el cual aumentó en aproximadamente un $12 \%(\mathrm{p}<0,05)$. En cuanto a los resultados de la escala de Ashworth modificada, se encontró que el promedio de puntuación en los extensores de rodilla izquierda se redujo de $2,75 \pm 0,6$ a $1,83 \pm 0,6$ y de $2,62 \pm 0,4$ a $1,81 \pm 0,2$ en extensores de rodilla derecha.

De manera similar, Rayegani et al. ${ }^{24}$ en un ensayo clínico prospectivo con 74 sujetos con LM en diferentes niveles de compromiso, $95 \%$ de los cuales eran varones y con edad media de 43 años, realizaron, durante 2 meses, una intervención con 3 sesiones diarias de PP con una duración de 20 minutos cada una, a una frecuencia no especificada. Los resultados obtenidos demostraron una disminución estadísticamente significativa en la espasticidad al finalizar los 2 meses de intervención, medida con la Escala de Ashworth modificada ( $p<0,05$ al comparar el grupo control sin PP y el experimental con PP). Además, utilizaron el reflejo $\mathrm{H}$, en el cual la razón de la amplitud máxima de la onda $\mathrm{H}$ (Hmax) respecto a la amplitud máxima de la onda $\mathrm{M}$ (Mmax) disminuyó en un $51 \%$ en el MI derecho $(\mathrm{p}<0,05)$ y un $50 \%$ en el MI izquierdo $(\mathrm{p}<0,05)$.
Se han propuesto dos mecanismos que permiten la disminución del tono muscular como respuesta al movimiento pasivo: 1) cambios en las propiedades mecánicas del tejido conectivo muscular y articular y 2) adaptación de la frecuencia de descarga del huso neuromuscular. Esto se puede evidenciar a través de la prueba del reflejo $\mathrm{H}$, que disminuye la amplitud de su onda (onda $\mathrm{H}$ ) debido a estos mecanismos fisiológicos ${ }^{25}$.

Efectos sobre el sistema musculoesquelético: los efectos del PP se han orientado al estudio de la densidad mineral ósea (DMO), rangos de movilidad articular y cambios en las proteínas musculares. En relación con $\mathrm{DMO}$, los estudios se han reportado únicamente en niños; esta ha sido evaluada con la absorciometría de rayos X de energía dual, DEXA o DXA. Johnston et al. ${ }^{26}$ en una serie de casos, investigaron en 4 niños con LM los efectos de un programa de pedaleo con estimulación eléctrica funcional comparado con PP a $50 \mathrm{rpm}, 1$ hora al día, 3 veces por semana durante 6 meses. En el grupo del PP encontraron que la DMO aumentó en uno de los niños $297,8 \%$ en la parte proximal del fémur, $23,8 \%$ a nivel distal del fémur y $39,2 \%$ en la tibia proximal; y en otro niño, 2,7\%, únicamente, en el fémur proximal. La diferencia de resultados entre los participantes fue explicada por los autores por la diferencia de edad, ya que el niño que presentó mayores cambios tenía 11 años y el otro 7. La diferencia de la DMO entre los segmentos en el participante con mejores resultados fue atribuida a la mayor carga que soporta el segmento óseo proximal durante la postura en sedente requerida durante el PP.

Lauer et al. ${ }^{27}$ investigaron en 30 niños 3 tipos de intervención: pedaleo con estimulación eléctrica funcional, PP y estimulación eléctrica de miembros inferiores durante 1 hora al día, 3 veces por semana durante 6 meses, con una frecuencia de $50 \mathrm{rpm}$. Se obtuvo, en el grupo del PP, un aumento de $29,2 \%$ en la DMO en fémur proximal; no se obtuvieron resultados positivos en el fémur distal y tibia proximal.

En relación con el rango de movimiento articular (ROM), Rayegani et al. ${ }^{24}$ encontraron un tamaño de efecto grande del PP (Cohen's d -1,71) en el incremento de los ROM de la cadera en los movimientos de abducción, aducción, flexión y extensión, y para el tobillo en dorsiflexión y plantiflexión, al culminar una intervención de 20 minutos del PP, realizada 3 veces al día durante 2 meses. Para la articulación de la rodilla, no se obtuvieron resultados significativos. Cabe mencionar que estos autores no reportan la frecuencia del pedaleo, 
solo indican que los participantes pedalearon en un ciclo predeterminado.

En cuanto a los cambios a nivel de proteínas musculares, Willoughby et al. ${ }^{28}$ sugieren que el PP tiene el potencial de atenuar la atrofia muscular, en razón a los hallazgos en un estudio pre-post con 8 personas con LM, quienes después de 24 sesiones de PP con una duración entre 20 a 90 minutos, donde la frecuencia de pedaleo fue aumentando hasta llegar al $75 \%$ de la FC máxima descrita para la edad de cada participante, reportaron un tamaño de efecto mediano (Cohen's $d=-0,79$ ) con un aumento de la expresión genética de proteínas de cadena pesada de miosina, asociadas con las fibras musculares de contracción rápida (MHC tipos IIa (49\%) y IIx (61\%)), y disminución de la actividad proteolítica asociada con la degradación muscular (UBI (45\%), E2 (68\%) y $20 \mathrm{~S}(96 \%)$, todos analizados $\mathrm{p}<0,05)$, medida a través de biopsia muscular. Los autores resaltan la importancia de sus resultados, ya que en personas con LM existe una degradación selectiva de los componentes miofibrilares, que comprenden la mayoría de las proteínas en el músculo, una atrofia severa como resultado de una mayor actividad de la vía proteolítica de ubiquitina, disminuciones en el área de la sección transversal de las fibras musculares y una mayor fatigabilidad causada por un aumento en el número de fibras tipo IIb.

En lo que respecta a las revisiones sobre los efectos del PP, Nardone et al. ${ }^{11}$ efectuaron una revisión de estudios experimentales tanto en animales (ratas) como en humanos, donde concluyen que, en los primeros, este modulaba el reflejo espinal y reducía la espasticidad, la disreflexia autonómica y provocaba efectos cardioprotectores; sin embargo, los estudios en sujetos humanos no lograron mostrar un efecto clínico en la reducción de la espasticidad y no respaldaron su aplicación para la prevención de complicaciones secundarias relacionadas con enfermedades cardiovasculares.

Phadke et al. ${ }^{14}$ por su parte, en una revisión que incluyó once artículos, indicaron que aproximadamente la mitad de los estudios de una sola sesión mostraron una mejora estadísticamente significativa en las respuestas agudas y que múltiples sesiones de PP mostraron beneficios en tres categorías: cardiovascular, musculoesquelético y neurológico, con tamaños de efecto moderado a grande.

\section{Conclusiones}

La revisión bibliográfica efectuada permitió identificar que los estudios del PP en personas con LM evalúan en su mayoría el efecto en una sesión; los efectos agudos cardiovasculares son los más estudiados. Los estudios que evaluaron efectos de más de una sesión tuvieron variables de análisis diferentes. Aunque algunos trabajos muestran un beneficio en los sistemas que evaluaron, otros tienden a no mostrar efectos.

Se evidencia que la literatura en el tema para los sujetos de interés en este estudio es escasa y hay una heterogeneidad clínica importante al comparar los parámetros de las intervenciones realizadas, las cuales varían en su duración desde los 5 hasta los 90 minutos y emplean cadencias de pedaleo que van entre 15 y 70 rpm, lo que hace que no sea posible abordar la consistencia en los resultados.

Adicionalmente, en la literatura en general no se encontraron publicaciones que hayan documentado los parámetros, incluidas las consideraciones biomecánicas, para el uso de esta herramienta de rehabilitación.

Finalmente, los autores de este artículo consideran que, aunque hay evidencia de efectos agudos benéficos en el funcionamiento de algunos sistemas con el uso del PP, aún se requieren nuevas investigaciones que aborden con mayor rigor los efectos a mediano y largo plazo de esta intervención en personas con lesión medular torácica.

\section{Referencias}

1. Bennett J, M Das J, Emmady PD. Spinal Cord Injuries. In: StatPearls. Treasure Island (FL): StatPearls Publishing; 2020. https://www.ncbi.nlm. nih.gov/books/NBK560721/

2. Huang H, Mao G, Chen L, Liu A. Progress and challenges with clinical cell therapy in neurorestoratology. J Neurorestoratol. 2015; 3: 9195. doi: https://doi.org/10.2147/JN.S74140

3. Chin L, King R, King M. Spinal cord injuries. Medscape. 2018. https://emedicine.medscape.com/ article/793582-overview.

4. Kirshblum SC, Burns SP, Biering-Sorensen F, Donovan $\mathrm{W}$, Graves DE, Jha A, et al. International standards for neurological classification of spinal cord injury (revised 2011). J Spinal Cord Med. 2011; 34(6): 535-546. doi: https://doi.org/10.1179/2045772 11X13207446293695

5. Kang Y, Ding H, Zhou HX, Wei ZJ, Liu L, Pan DY, et al. Epidemiology of worldwide spinal cord injury: a literature review. J Neurorestoratol. 2018; 6: 1-9. doi: https://doi.org/10.2147/JN.S143236

6. Carvajal C, Pacheco C, Gómez C, Calderón J, Cadavid C, Jaimes F. Características clínicas y 
demográficas de pacientes con trauma raquimedular. Acta Med. Colomb. 2015; 40(1): 45-50. doi: https:// doi.org/10.36104/amc.2015.403

7. Cragg JJ, Noonan VK, Krassioukov A, Borisoff J. Cardiovascular disease and spinal cord injury: results from a national population health survey. Neurology. 2013; 81(8): 723-728. doi: https://doi.org/10.1212/ WNL.0b013e3182a1aa68

8. Sezer N, Akkuş S, Uğurlu FG. Chronic complications of spinal cord injury. World J Orthop. 2015; 6(1): 2433. doi: https://dx.doi.org/10.5312/wjo.v6.i1.24

9. Hagen EM. Acute complications of spinal cord injuries. World J Orthop. 2015; 6(1):17-23. doi: https://dx.doi.org/10.5312/wjo.v6.i1.17

10. Gaspar R, Padula N, Freitas TB, de Oliveira JPJ, Torriani-Pasin C. Physical exercise for individuals with spinal cord injury: Systematic review based on the international classification of functioning, disability, and health. J Sport Rehabil. 2019; 28(5): 505-516. doi: https://doi.org/10.1123/jsr.2017-0185

11. Nardone R, Orioli A, Golaszewski S, Brigo F, Sebastianelli L, Höller Y, Frey V, et al. Passive cycling in neurorehabilitation after spinal cord injury: A review. J Spinal Cord Med. 2017; 40(1): 8-16. doi: https://doi.org/10.1080/10790268.2016.1248524

12. O’Sullivan S, Schmitz T, Fulk G. Physical Rehabilitation. $6^{\text {th }}$ ed.: F.A. Davis Company, Philadelphia. 2013.

13. Stokes M, Stack E. 2013. Lesión de la médula espinal: Fisioterapia en la rehabilitación neurológica. $3^{\mathrm{a}}$ ed.: Elsevier, Madrid.

14. Phadke CP, Vierira L, Mathur S, Cipriano G, Jr., Ismail F, Boulias C. Impact of passive leg cycling in Persons with spinal cord injury: A Systematic Review. Top Spinal Cord Inj Rehabil. 2019; 25(1): 83-96. doi: https://doi.org/10.1310/sci18-00020

15. Johnston T. Biomechanical considerations for cycling interventions in rehabilitation. Phys Ther. 2007; 87: 1243-1252. doi: https://doi.org/10.2522/ ptj.20060210

16. Muraki S, Ehara Y, Yamasaki M. Cardiovascular responses at the onset of passive leg cycle exercise in paraplegics with spinal cord injury. Eur J Appl Physiol. 2000; 81(4): 271-274. doi: https://doi. org/10.1007/s004210050042

17. Muraki S, Tsunawake N. Relationship between pedaling rate and physiological responses during passive leg cycling. Isokinet Exerc Sci. 2008; 16: 1924. doi: https://doi.org/10.3233/ies-2008-0291

18. Ballaz L, Fusco N, Crétual A, Langella B, Brissot R. Peripheral vascular changes after home-based passive leg cycle exercise training in people with paraplegia: a pilot study. Arch Phys Med Rehabil. 2008;
89(11): 2162-2166. doi: https://doi.org/10.1016/j. apmr.2008.04.018

19. Ballaz L, Fusco N, Cretual A, Langella B, Brissot R. Acute peripheral blood flow response induced by passive leg cycle exercise in people with Spinal cord injury. Arch Phys Med Rehabil. 2007; 88(4): 471476. doi: https://doi.org/10.1016/j.apmr.2007.01.011

20. Groothuis JT, Hopman MT. Does passive cycling induce changes in peripheral blood flow in persons with spinal cord injury? Arch Phys Med Rehabil. 2007; 88(12): 1740-1741. doi: https://doi. org/10.1016/j.apmr.2007.09.021

21. Ter Woerds W, De Groot PC, van Kuppevelt DH, Hopman MT. Passive leg movements and passive cycling do not alter arterial leg blood flow in subjects with spinal cord injury. Phys Ther. 2006; 86(5): 636645. doi: https://doi.org/10.1093/ptj/86.5.636

22. Kakebeeke T, Lechner H, Knapp P. The effect of passive cycling movements on spasticity after spinal cord injury: preliminary results. Spinal Cord. 2005; 43: 483-488. doi: https://doi.org/10.1038/ sj.sc. 3101747

23. Krause P, Szecsi J, Straube A. Changes in spastic muscle tone increase in patients with spinal cord injury using functional electrical stimulation and passive leg movements. Clin Rehabil. 2008; 22(7): 627-634. doi: https://doi.org/10.1177/0269215507084648

24. Rayegani SM, Shojaee H, Sedighipour L, Soroush MR, Baghbani M, Amirani OB. The effect of electrical passive cycling on spasticity in war veterans with spinal cord injury. Front Neurol. 2011; 2: 39. doi: https://doi.org/10.3389/fneur.2011.00039

25. Pérez-Parra JE, Henao-Lema CP. Efecto de la movilización articular sobre la amplitud del Reflejo $\mathrm{H}$ en personas con espasticidad. Rev Cienc Salud. 2011; 9(2):125-140

26. Johnston TE, Smith BT, Oladeji O, Betz RR, Lauer RT. Outcomes of a home cycling program using functional electrical stimulation or passive motion for children with spinal cord injury: a case series. J Spinal Cord Med. 2008; 31(2): 215-221. doi: https:// doi.org/10.1080/10790268.2008.11760715

27. Lauer RT, Smith BT, Mulcahey MJ, Betz RR, Johnston TE. Effects of cycling and/or electrical stimulation on bone mineral density in children with spinal cord injury. Spinal Cord. 2011; 49(8): 917923. doi: https://doi.org/10.1038/sc.2011.19

28. Willoughby DS, Priest JW, Jennings RA. Myosin heavy chain isoform and ubiquitin protease mRNA expression after passive leg cycling in persons with spinal cord injury. Arch Phys Med Rehabil. 2000; 81(2): 157-163. doi: https://doi.org/10.1016/S00039993(00)90134-5 